\title{
TIPOLOGIA DO CONSUMIDOR DE FRUTOS DO CERRADO
}

Typology of the Consumer of Cerrado Fruits

Tipología del Consumidor de Frutos del Cerrado

\section{Camila Mariane da Silva Soares ${ }^{* 1,2}$, Aynaran Oliveira de Aguiar ${ }^{1}$, Romilda Ramos da Silva $^{1}$, Andreia Ibiapina ${ }^{1}$, André Leonardo dos Santos $^{2}$, Glêndara Aparecida de Souza Martins ${ }^{1}$}

${ }^{1}$ Laboratório de Cinética e Modelagem de Processos, Engenharia de Alimentos, Universidade Federal do Tocantins, Palmas, TO, Brasil.

${ }^{2}$ Discente do Mestrando em Ciências e Tecnologia de Alimentos, Universidade Federal do Tocantins, Palmas, TO, Brasil.

*Correspondência: Laboratório de Cinética e Modelagem de Processos, Universidade Federal do Tocantins, Av. NS 15, ALCNO 14 - Bloco II, Sala 06, 109 Norte, Palmas, Tocantins, Brasil. CEP:77.010-090. e-mail camilamariane@mail.uft.edu.br

Artigo recebido em 05/08/-2019 aprovado em 17/06/2019 publicado em 02/10/2019.

\section{RESUMO}

O Cerrado é um bioma altamente diversificado, rico em frutas que possuem sabor sui-generis, podendo ser consumidas in natura ou processada. A maioria desses frutos são ricos em vitaminas, antocianinas, flavonóides e possuem alto potencial antioxidante. No entanto ainda há pouco conhecimento da população quanto aos benefícios proporcionados pelo consumo, potencial econômico e aplicação tecnológica desses frutos. Com base nisso, essa pesquisa foi desenvolvida com o intuito de fazer um levantamento sobre o conhecimento da população em relação aos frutos nativos do Cerrado, além de verificar o perfil dos consumidores e o conhecimento sobre os benefícios desses frutos. Foram entrevistadas 352 pessoas de todos os estados brasileiros, que responderam a um questionário online. Constatou-se, que $91 \%$ possui conhecimento quanto aos frutos, no entanto 55,3\% não tinham ciência quanto aos benefícios de consumo e 12,2\% nunca consumiram, mesmo os residentes das regiões do Norte e Nordeste, que abrangem o bioma do Cerrado. Concluiu-se que a maioria dos consumidores de frutos do cerrado são mulheres, sendo cajuí, buriti e murici os frutos mais populares, por já estrem inclusos na alimentação da maioria dos entrevistados. No entanto ainda é vasto o desconhecimento dos consumidores quanto a diversidade dos frutos e potencial de processamento.

Palavras-chave: Aplicação tecnológica; Benefícios; Pesquisa exploratória;

\section{ABSTRACT}

The Cerrado is a highly diversified biome, rich in sui generis tasting fruits which can be consumed in natura or in a processed form. A majority of these fruits are rich in vitamins, anthocyanins, flavonoids and possess great antioxidant power. However, there still exists a lack of knowledge from the general population regarding the benefits of consumption, economic potential and technological application of these fruits. Based on this, this research was developed with the purpose of carrying out a survey regarding the population's knowledge regarding the native fruits of the Cerrado, besides checking the profile of the consumers and their knowledge in respect to the benefits of the fruits. We interviewed 352 people from all Brazilian states, who answered an online questionnaire. It was observed that $91 \%$ have knowledge about the fruits, however 55,3\% were not aware of the benefits of consumption and $12.2 \%$ never consumed, even the residents of the North and Northeast regions, which cover the Cerrado biome. It was concluded that the majority of consumers of fruits of the cerrado are women, being cajui, buriti and died the most 
popular fruits, already included in the diet of most of the interviewees. However, there is still a great deal of consumer ignorance about the diversity of fruits and processing potential.

Keywords: Technological application; Benefits; Exploratory research;

\section{RESUMEN}

El Cerrado es un bioma altamente diversificado, rico en frutas que tienen sabor sui-generis, pudiendo ser consumidas in natura o procesada. La mayoría de estos frutos son ricos en vitaminas, antocianinas, flavonoides y poseen alto potencial antioxidante. Sin embargo, todavía hay poco conocimiento de la población en cuanto a los beneficios proporcionados por el consumo, potencial económico y aplicación tecnológica de esos frutos. Con base en ello, esta investigación fue desarrollada con el propósito de hacer un relevamiento sobre el conocimiento de la población en relación a los frutos nativos del Cerrado, además de verificar el perfil de los consumidores y el conocimiento sobre los beneficios de esos frutos. Se entrevistaron a 352 personas de todos los estados brasileños, que respondieron a un cuestionario en línea. Se constató que el $91 \%$ tiene conocimiento de los frutos, sin embargo, el 55,3\% no tenía conocimiento de los beneficios de consumo y el 12,2\% nunca consumió, incluso los residentes de las regiones del Norte y Nordeste, que cubren el bioma del bioma, Cerrado. Se concluyó que la mayoría de los consumidores de frutos del cerrado son mujeres, siendo cajuí, buriti y murici los frutos más populares, por ya estrem incluidos en la alimentación de la mayoría de los entrevistados. Sin embargo, sigue siendo el desconocimiento de los consumidores en cuanto a la diversidad de los frutos y el potencial de procesamiento.

Descriptores: Aplicación tecnológica; beneficios; Investigación exploratoria;

\section{INTRODUÇÃO}

O Cerrado é considerado um dos biomas mais ricos em diversidade do mundo, sendo o segundo maior no Brasil, ocupando cerca de $22 \%$ do território nacional, onde $90 \%$ está localizado nos estados de São Paulo, Minas Gerais, Mato Grosso, Goiás e Bahia (SILVA et al., 2017) e Tocantins. Nele são encontradas inúmeras espécies de plantas nativas que possuem características sensoriais particulares, que podem ser utilizadas na alimentação humana tanto in natura como após o processamento, o que as tornam um grande potencial de exportação nacional e internacional, aumentando assim o interesse da população e das indústrias por inovação, proporcionando competitividade no mercado (SCARIOT; RIBEIRO, 2015; MORZELLE et al., 2015).

Esse bioma apresenta diversidade ampla de plantas frutíferas, intra e interespecíficas com bastante potencial de uso agrícola e tecnológico (ALVES et al., 2014). No entanto ainda há pouco conhecimento da população quanto aos benefícios proporcionados pelo consumo dos frutos in natura ou processados (AGUIAR, 2018). Os frutos nativos são ricos em compostos fenólicos, dentre eles, flavonóides, antocianinas, taninos e compostos fenólicos simples os quais representam a principal classe de compostos bioativos (BAILÃO et al., 2015). Com base nesse contexto o estudo sobre o perfil e conhecimento do consumidor é importante, pois pode contribuir no entendimento de como são tomadas as decisões sobre o consumo, compra e também no processo de geração de valor para o consumidor (PEROSA et al., 2012). Desta forma, o presente estudo teve como objetivo fazer um levantamento em todos estados brasileiro sobre o conhecimento dos frutos nativos do Cerrado, além de verificar o perfil dos consumidores e o conhecimento sobre os benefícios desses frutos.

\section{MATERIAIS E MÉTODOS}

O método utilizado nesta pesquisa foi exploratório. As informações foram coletadas por intermédio de um questionário estruturado com 
perguntas objetivas. $\mathrm{O}$ mesmo foi aplicado durante o mês de setembro de 2018, de forma online. O questionário abrangeu 352 participantes, de diferentes estados brasileiros, sexo, faixa etária, classe social e escolaridade. Os dados recolhidos foram tabulados no software Microsoft Excel, submetidos à estatística descritiva e esquematizados em gráficos.

\section{RESULTADOS E DISCUSSÃO}

Foram analisados 352 participantes de ambos os sexos, havendo representantes de todas as regiões brasileiras, $69 \%$ da região Norte, $15 \%$ da região Nordeste, e as regiões Centro-Oeste, Sul e Sudeste representam $6 \%, 3 \%$ e $4 \%$, respectivamente. Os entrevistados tinham a faixa etária entre 25 e 34 anos (45\%), seguida por 19 e 24 anos (30\%), 35 e 50 anos (14\%), maiores que 50 (8\%) e até 18 anos (4\%).

Baseado no que foi relatado pelos entrevistados constatou-se, que $91 \%$ possui conhecimento quanto aos frutos oriundos do Cerrado brasileiro, $63 \%$ são do sexo feminino e $53 \%$ destas afirmam que consomem frutos oriundos do cerrado (Figura 1).

Figura 1: Consumo de acordo com o gênero.

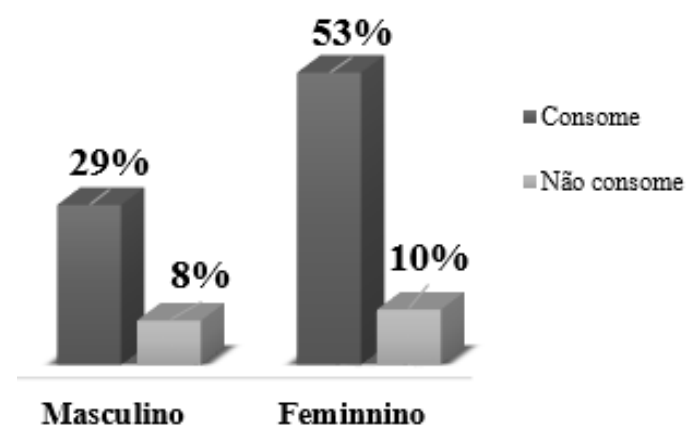

Quando questionados sobre a frequência de consumo desses frutos, $41 \%$ dos entrevistados afirmam consumir uma vez a cada semana, $23 \%$ duas a três vezes na semana, $18 \%$ nunca consumiram, $13 \%$ diariamente e $5 \%$ afirmaram que consomem raramente. Observou-se também a predominância do gênero feminino quanto à frequência de consumo, onde $25 \%$ diz consumir pelo menos uma vez a cada semana (Figura 2). Segundo Farias et al (2014) e Perez et al (2008), a mulher ainda é a maior responsável pela compra e consumo de frutas e hortaliças.

Figura 2: Frequência de consumo de frutos do cerrado acordo com o gênero.
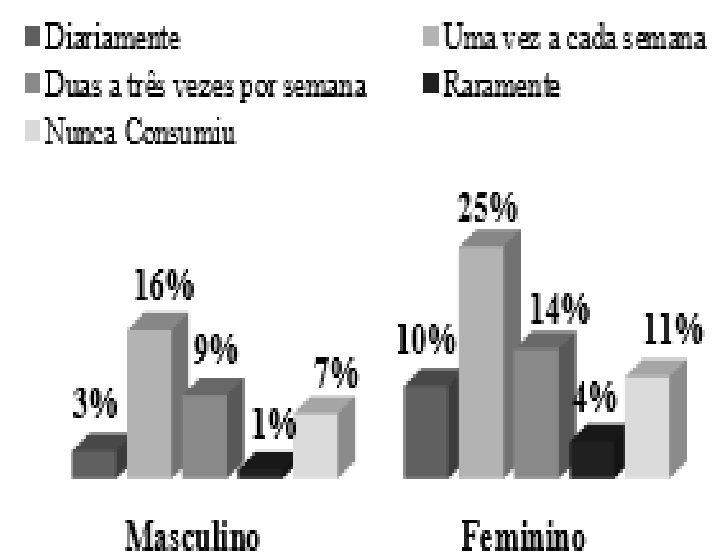

O nível econômico representado pela renda mensal, foi de $44 \%$ com até 2 salários mínimos, $41 \%$ de 2 a 5 salários os outros $15 \%$ acima de 5 salários, sendo possível observar que os maiores consumidores são os que possuem uma renda de até 2 salários mínimos com faixa etária entre 19 a 34 anos.

Quanto ao grau de escolaridade dos entrevistados, 29,7\% está cursando Ensino superior, $29,4 \%$ possui graduação completa, e $22,8 \%$ pósgraduação. Percebeu-se que quanto menor o grau de escolaridade maior a frequência e consumo destes frutos. Farias et al. (2014), descreve que o nível educacional e renda familiar, são fatores determinantes para uma maior ou menor ingestão de frutas, além disso Perosa et al. (2012), destaca que parâmetros como sabor, local de aquisição e preço, influenciam no ato da compra.

No que diz respeito aos locais de obtenção dos frutos, $39 \%$ dos entrevistados afirmaram adquiri-los em feiras livres, $33 \%$ tem como fonte principal o próprio Cerrado, $25 \%$ dos entrevistados compram em 
supermercados e 3\% em lojas de produtos orgânicos. Geralmente esses frutos, são amplamente encontrados na natureza, nas regiões do Cerrado, assim como em feiras livres (GUÉNEAU et al., 2017), o que corrobora com o resultado encontrado nesta pesquisa.

Os frutos mais populares entre os entrevistados foram o cajuí $(68,6 \%)$, seguido pelo buriti (68,3\%), e murici com 48,2\% (Figura 4). Esse resultado mostra como a diversidade dos frutos oriundos do Cerrado ainda é pouco conhecida e divulgada entre a população brasileira, pois frutos como guapeva, araticum e gabiroba, por exemplo, são encontrados, em determinadas épocas, em abundância no Cerrado brasileiro, no entanto ainda não tem seu potencial totalmente explorado, devido à falta de conhecimento quanto às suas características sensoriais e nutricionais.

Figura 4. Conhecimento dos entrevistados sobre os frutos do Cerrado.

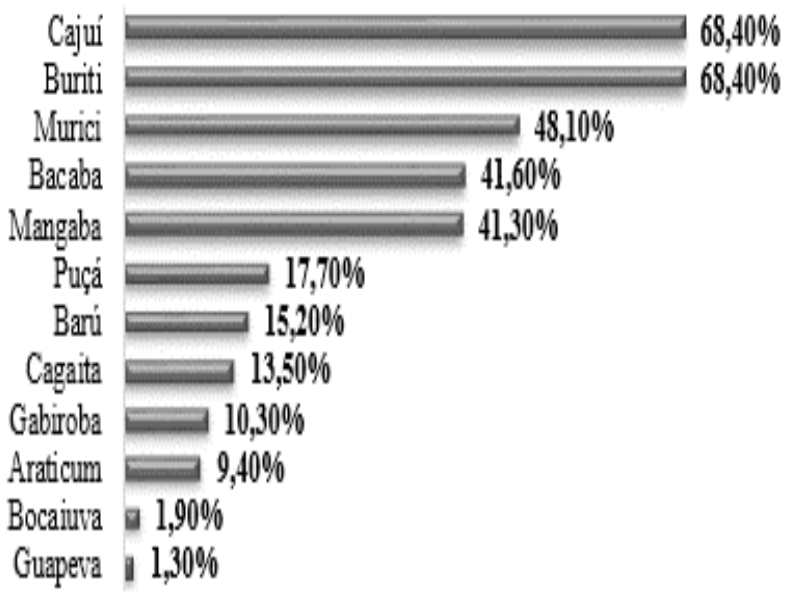

Sobre a forma de consumo destes frutos (Figura 5), verificou-se que a maioria dos entrevistados consomem esses frutos através de sucos $(84,8 \%)$, seguida por picolés $(60,1 \%)$. O processamento de frutos é uma atividade bastante importante para a conservação de alimentos, pois aumentam a vida útil do produto diminuindo a probabilidade proliferação de microrganismos, pois geralmente esse processamento envolve a retirada da quantidade de água do fruto como no caso do processamento de frutas secas, doces e polpas concentradas. Propiciando ao consumidor o acesso a esses frutos durante todo o ano, uma vez que grande parte deles apresenta frutificação em períodos específicos (ARRUDA et al., 2014; LEONEL et al., 2014; SAINZ et al., 2015; SIQUEIRA et al., 2018).

Figura 5. Conhecimento dos entrevistados quanto as formas processadas dos frutos do cerrado

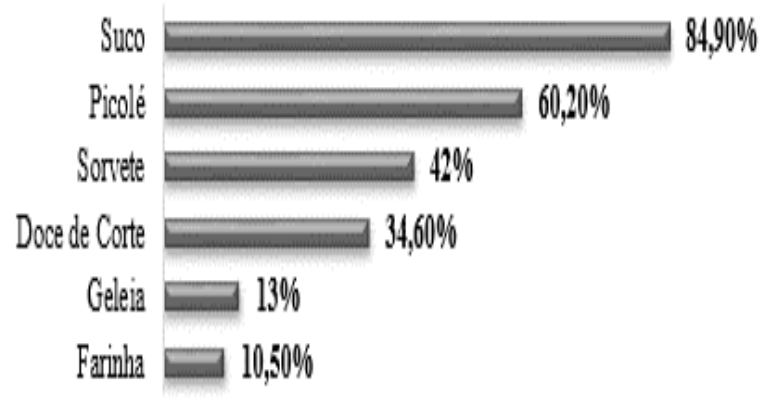

Esses frutos, além de possuírem alto teor nutricional, proporcionam sabor único e característico, que podem ser empregados nas indústrias de alimentos para a fabricação de sucos, geleias, doces, polpas e sorvetes. Entretanto dentre os frutos citados acima poucos são comercialmente explorados e isso acarreta em grandes perdas dos frutos, que poderiam ser processados e transformados em diferentes produtos (SILVA et al., 2018).

Embora $84 \%$ dos entrevistados tenham declarado residência nas regiões Norte e Nordestes, regiões com grandes áreas de Cerrado, 55,3\% desse total declararam não possuir conhecimento sobre os benefícios gerados pelo consumo e 12,2\% nunca consumiu esses frutos. Em sua maioria os frutos oriundos do Cerrado brasileiro, são fontes de propriedades funcionais benéficas a saúde dos consumidores (SIQUEIRA et al., 2013; MORZELLE et al., 2015), porém, boa parte ainda não são conhecidas e consumidas, como indica a pesquisa. Para Oliveira et al. (2017), esse resultado mostra como 
é necessário que produtores e a população local, por meio da extensão rural promovam a valorização e divulgação do Cerrado brasileiro.

\section{CONCLUSÃO}

Com base no estudo, concluiu-se que a maioria dos consumidores de frutos oriundo do cerrado são mulheres, e os frutos mais consumidos foram, cajuí, buriti e murici, pois já estão inclusos na alimentação de grande parte dos entrevistados. No entanto os resultados demonstram o desconhecimento dos consumidores quanto a diversidade dos frutos devido ao seu potencial pouco explorado quanto ao processamento. Baseado nisto, pode-se afirmar que a industrialização desses frutos facilitaria o conhecimento da população de um modo geral e consequentemente aumentaria o consumo.

Dessa forma, os dados obtidos através do questionário são fundamentais para o planejamento de estratégias incentivadoras de consumo desses produtos com ênfase nas vantagens relacionadas à saúde e aproveitamento da flora nativa da região do Cerrado.

\section{AGRADECIMENTO}

Ao LaCiMP- Laboratório de cinética e modelagem de processos pelo apoio no desenvolvimento da pesquisa

\section{REFERÊNCIAS}

AGUIAR, A. O. D. Processamento e aproveitamento do fruto do araticum (Annona Crassiflora Mart.) em forma de doce em massa (Dissertação de mestrado). Universidade Federal do Tocantins, Palmas-TO, 2018.

ALVES, A. M., FERNANDES, D. C., DE OLIVEIRA SOUSA, A. G., NAVES, R. V., \& NAVES, M. M. V. Physical and nutritional characteristics of pequi fruits from Tocantins, Goiás and Minas Gerais States. Brazilian Journal of Food Technology, v. 17, n. 3, p. 198, 2014.

ARRUDA, H. S., BOTREL, D. A., DE BARROS FERNANDES, R. V., \& ALMEIDA, M. E. F. Ação extensionista para fortalecer a importância alimentar e ambiental dos frutos do cerrado em Rio Paranaíba (MG). Revista Conexão UEPG, v. 10, n. 2, p. 264277, 2014.

AVIDOS, M. F. D., \& FERREIRA, L. T. Frutos dos Cerrados. Biotecnologia Ciência e Desenvolvimento, v. 3 , n. 15 , p. $36-41,2000$.

BAILÃO, E. F. L. C., DEVILLA, I. A., DA CONCEIÇÃO, E. C., \& BORGES, L. L. Bioactive compounds found in Brazilian Cerrado fruits. International journal of molecular sciences, v. 16, n. 10, p. 23760-23783, 2015.

FARIAS, R. DE M. BARRETO, C. F., ZANDONÁ, R. R., ROSADO, J. P., MARTINS, C. Comportamento do consumidor de frutas na região da fronteira oeste do Rio Grande do Sul com Argentina e Uruguai ${ }^{1}$. Embrapa Clima Temperado-Artigo em periódico indexado (ALICE), 2014.

GUÉNEAU, S., DINIZ, J. D. D. A. S., MENDONÇA, S. D., \& GARCIA, J. P. Construção social dos mercados de frutos do Cerrado: entre sociobiodiversidade e alta gastronomia. Século XXIRevista de Ciências Sociais, v. 7, n. 1, p. 130-156, 2017.

LEONEL, S., LEONEL, M., \& SAMPAIO, A. C. Processamento de frutos de abacaxizeiro cv smooth cayenne: perfil de açúcares e ácidos dos sucos e composição nutricional da farinha de cascas. Revista Brasileira de Fruticultura, v. 36, n. 2, p. 433-439, 2014.

MORAIS, E. C. D., PATIAS, S. G. D. O., FERREIRA, N. S. D. S., PICANÇO, N. F. M., RODRIGUES, E. C., NASCIMENTO, E., \& FARIA, R. A. P. G. D. Bioactive compounds and physicochemical characteristics of in natura and pasteurized araticum pulp. Brazilian Journal of Food Technology, v. 20, e2016142, 2017.

MORZELLE, M. C., BACHIEGA, P., DE SOUZA, E. C., BOAS, E. V. D. B. V., \& LAMOUNIER, M. L. Caracterização química e física de frutos de curriola, gabiroba e murici provenientes do cerrado brasileiro. Revista Brasileira de Fruticultura, v. 37, n. 1, p. 96103, 2015.

OLIVEIRA, P. H. B., XAVIER, E. G., \& CRISTINA DE ARAUJO, A. Perfil dos consumidores de frutos do cerrado no município de goianésia. In CONGRESSO INTERDISCIPLINAR RESPONSABILIDADE, CIÊNCIA E ÉTICA, 4, Goianésia, Anais. FACEG, v. 4, n. 1, 2017. 
PEREZ, R., RAMOS, A. M., BINOTI, M. L., SOUSA, P. H. M., MACHADO, G. M., \& CRUZ, I. B. Perfil dos consumidores de hortaliças minimamente processadas de Belo Horizonte. Horticultura Brasileira, v. 26, n. 4, p. 441-446, 2008.

PERFEITO, D. G. A., CORRÊA, I. M., \& PEIXOTO, N. Elaboração de bebida com extrato hidrossolúvel de soja saborizada com frutos do cerrado. Revista de agricultura neotropical, v. 4, n. 1, p. 21-27, 2017.

PEROSA, J. M. Y., TARSITANO, M. A. A., MARTINS, M. I. E. G., PIGATTO, G., \& ANTONANGELO, A. Perfil do consumidor de frutas em cidades do interior do estado de São Paulo-SP. Revista Brasileira de Fruticultura, v. 34, n. 4, p. 1084-1090, 2012.

PEROSA, J. M. Y., TARSITANO, M. A. A., MARTINS, M. I. E. G., PIGATTO, G., \& ANTONANGELO, A. Perfil do consumidor de frutas em cidades do interior do estado de São PauloSP. Revista Brasileira de Fruticultura, p. 10841090, 2012.

SAINZ, R. L., \& DA SILVA VENDRUSCOLO, J. L. Propriedades da poligalacturonase e pectinametilesterase em pêssegos [Prunus persica (L.) Batsch] de cultivares brasileiras. Revista Brasileira de Tecnologia Agroindustrial, v. 9, n. 1, 2015.

SCARIOT, A., \& RIBEIRO, J. F. Boas práticas de manejo para o extrativismo sustentável da Cagaita. 1 ed., Brasília. Embrapa Recursos Genéticos e Biotecnologia, 2015.
SILVA, C. D. M., PIRES, C. R. F., LIMA, J. P., PEREIRA, A. S., \& SILVA, C. A. Osmotic dehydration to obtain cagaita raisins. Journal of bioenergy and food science, v. 2, n. 4, p. 226-233, 2015.

SILVA, E. P. D., ABREU, W. C. D., GONÇALVES, O. A., DAMIANI, C., BOAS, V., \& DE BARROS, E. V. Characterization of chemical and mineral composition of marolo (Annona crassiflora Mart) during physiological development. Food Science and Technology, v. 37, n. 1, p. 13-18, 2017.

SILVA, J. C. F., SOUZA, I. A., CONCEIÇÃO, D. R., CORDEIRO, C. A. S., MACHADO, A. S., \& RIOS, A. D. F. Desenvolvimento inicial do cajuzinho do cerrado. Ipê Agronomic Journal, v. 2, n. 1, p. 23-30, 2018.

SIQUEIRA, A. P. S., MORGADO, C. M. A., CAVALCANTE, K. A., JÚNIOR, L. C. C., \& SOUZA, E. R. B. Vida útil de variedades de mangaba endêmicas do cerrado em diferentes estádios de maturação. Journal of neotropical agriculture, v. 5, n. 3, p. 91-96, 2018.

SIQUEIRA, E.M. A., ROSA, F. R., FUSTINONI, A. M., SANTANA, L. P., ARRUDA, S. F. Brazilian savanna fruits contain higher bioactive compounds content and higher antioxidant activity relative to the conventional red delicious apple. Plos One Cambridge, v. 8, n. 8, p. e72826, 2013. 